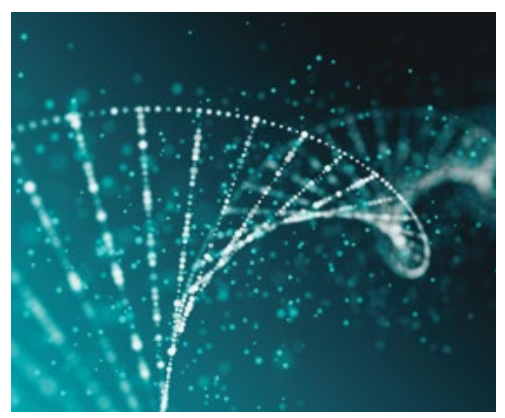

\title{
A glimpse of 21st century care
}

\section{Steve Hambleton}

\section{Background}

Navigating Australia's health system is made more complex by its siloed origins and multi-sourced funding environment.

\section{Objectives}

This article seeks to illustrate the benefits of the emerging digital health system by reference to typical patients that Australian general practitioners are treating.

\section{Discussion}

Australia must move towards connected, patient-centred care, in alignment with the principles found within the report from the Primary Health Care Advisory Group and Australia's National Digital Health Strategy.
EVER SINCE John Howard's time as prime minister, with each 'Intergenerational Report' we are reminded about the increasingly ageing population ${ }^{1}$ and that Australians are increasingly living with chronic conditions such as heart disease, diabetes, cancers, respiratory diseases and mental illness. ${ }^{2}$ We know that there is significant waste and duplication within our system, and we know that 2-3\% of hospital admissions are potentially preventable, with significant numbers due to medication misadventure. ${ }^{3}$ Surely in the 21 st century we can do better.

To complicate and frustrate matters further, we have complex funding systems that support the various silos that make up our health system. Figure 1, reproduced from Flying blind: Australian consumers and digital health, ${ }^{4}$ shows funders, regulators and their complex inter-relationships. Finding the consumer in Figure 1 is similar to a game of 'Where's Wally?'

As successive health ministers have come in to their portfolios, they have been further informed about the looming health funding crisis that Australia is facing, and all have tried various initiatives to steer the health system into calmer waters. In the past few years, governments have realised that the only way forward is to make the health system more consumercentric. Some of those initiatives are now beginning to mature, and it is possible that we are seeing a glimpse of what 21st century medicine might look like.

In 2003, federal, state and territory health ministers established the National eHealth Transition Authority
(NEHTA) to develop the specifications, standards and infrastructure necessary for an interconnected health sector, select a common language for health communications and create unique healthcare identification numbers for all individuals, providers and organisations. ${ }^{5}$

On that platform, on 1 July 2012, NEHTA launched the 'opt-in' Personally Controlled Health Record, now called My Health Record (MyHR), to share summary health information with all registered providers. MyHR supports the healthcare of nearly six million citizens and has moved to 'opt-out' status, so by the end of 2018 it will support a critical mass of participants. In addition, with progressively more information flowing into it, MyHR will soon contain useful clinical information for patients who most need it. The 'critical mass' of participants and information about them drove the success of the MyEHR project in the Northern Territory. ${ }^{6}$

The move to opt out is expected to bring forward the benefits of e-health, including reduced duplication of tests, better coordination of care for people with chronic and complex conditions, less medication misadventure, and betterinformed treatment decisions through improved sharing of information.

In addition to those system benefits, sharing information directly with our patients is a key building block to support 'activation of our patients' to deliver against key recommendation 3 of the Primary Health Care Advisory Group (PHCAG) report: Activate patients to be 
engaged in their care. ${ }^{2}$ Relevant information within MyHR can support and encourage patients and their carers to be more active and effective partners in their healthcare. To assist patients with their care decisions, MyHR is available on home computers and mobile devices through trusted third-party providers using the secure MyGov portal. ${ }^{7}$ An increasing body of research is showing that improving patient activation has great potential to improve health outcomes. ${ }^{8}$

The opt-out option has raised concerns about security and privacy, particularly with high-profile data breaches in social media and in the Singapore SingHealth system. ${ }^{9}$

To be clear, the system in Australia is built to the highest security standards, with active and passive security monitoring and penetration testing. Providers (including hospitals) need to have individual and organisation identifiers and conformant software loaded with encryption security keys to access the record. Even then, they can only access patients who are already registered on their clinical information systems. Every access to every health record is logged and visible to the consumer, who has control over what goes there. It is not their whole history; it is an agreed subset of their history. Consumers can remove records and set access codes to the entire record or to particular documents. No one can see those records without the pin supplied by the consumer, unless there is an emergency and they are unable to communicate. Consumers can also set up alerts via text message or email if their record is accessed or changed. ${ }^{10}$

I have worked in my practice for just over thirty years, assisted by computerisation in the past twenty. Being a large group practice, I have tried to progressively keep the practice health summary up to date as new information arrived. My summaries include current medication and allergies, a list of both current and past medical problems and key results, including endoscopy reports, echocardiography reports and some radiology reports where they are deemed to be clinically important. Those

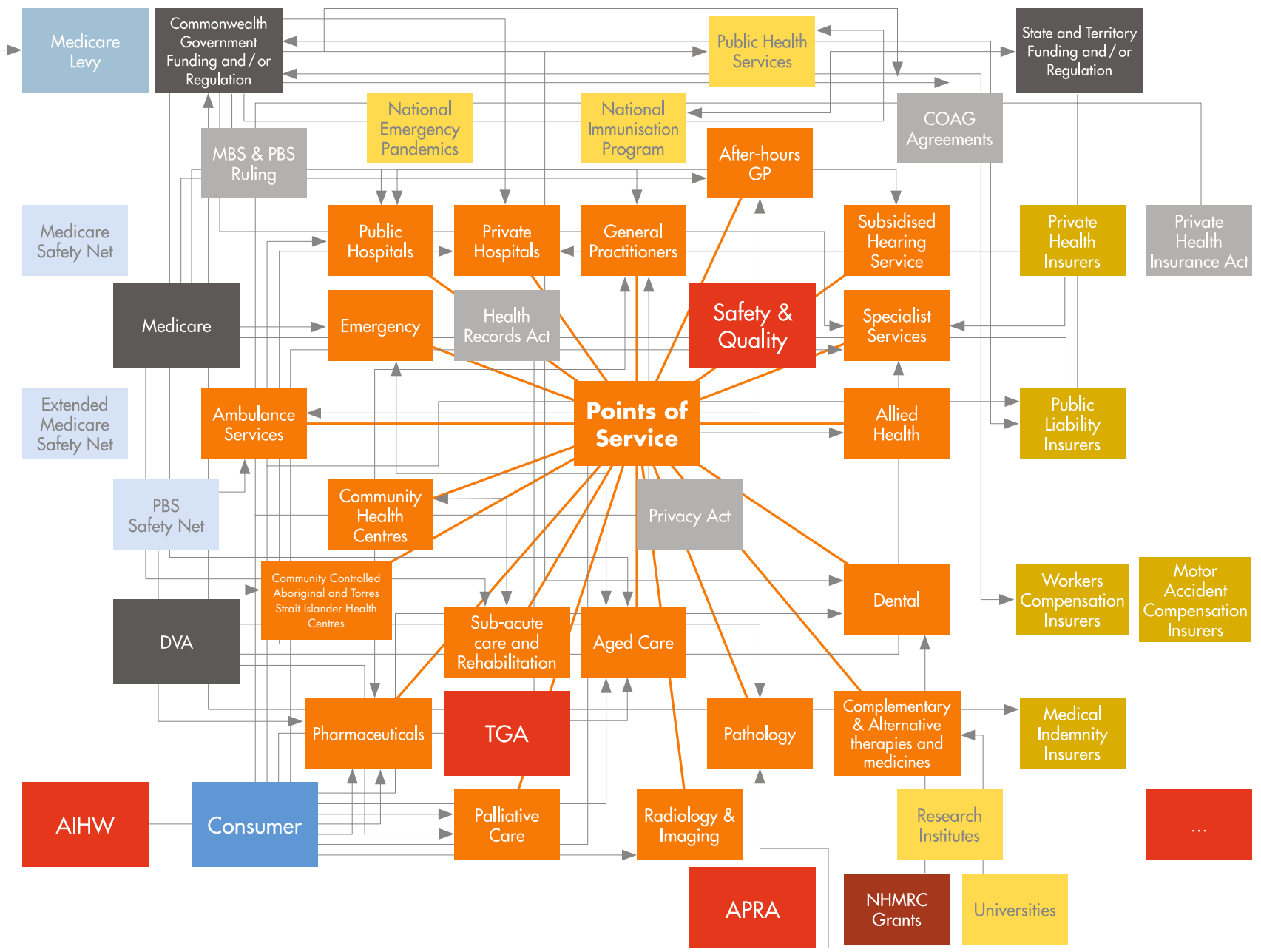

Figure 1. The Australian healthcare environment

AIHW, Australian Institute of Health and Welfare; APRA, Australian Prudential Regulation Authority; COAG, Council of Australian Governments; DVA, Department of Veterans' Affairs; GP, general practitioner; MBS, Medicare Benefits Schedule; NHMRC, National Health and Medical Research Council; PBS, Pharmaceutical Benefits Scheme; TGA, Therapeutic Goods Administration 
summaries also form the basis of my care plans, keeping both the patient and their healthcare team fully informed. Since 2014, I have begun sharing that practice health summary with the patients' MyHR, and I have been actively encouraging my patients and my medical neighbourhood to interact with MyHR, with some very encouraging results.

On one occasion, a patient aged 73 years informed me that he had logged in to his MyHR at home, reviewed his medication list on the latest health summary and concluded that, 'It was wrong!' We then went through the list of medications, and I explained to him the meanings of the Latin abbreviations. It was obvious to only the pharmacist and myself that prn, pro re nata or as required, was very different to pr, per rectum, and that bd, bis de sumendum, was twice daily and not 'bed time', as the patient thought. That was the day I decided to stop using Latin abbreviations and resolved to change all my directions to longhand English.

Another glimpse of the future is revealed when I reflect on another one of my complex patients. He was aged 65 years and his medical history included longstanding dilated cardiomyopathy and heart failure, a defibrillating pacemaker, insulin-dependent diabetes mellitus, morbid obesity and stage 3 chronic kidney disease.

After starting a new medication prescribed by his cardiologist, the patient reported that he was warned to have regular blood tests to monitor his renal function. When I asked the patient, whose wife was also in attendance on both occasions, for the name of the medication, they were unable to recall it. The letter from the cardiologist had not yet arrived. General practitioners (GPs) and general practice nurses will recognise this familiar scenario.

I noted that it was unlikely that the cardiologist had software that could upload an event summary or a specialist letter. The patient attended a pharmacy that had not yet upgraded their software to be MyHR-compatible, so there was no dispensed record. I hoped that the Pharmaceutical Benefits Scheme claims data for that medication were recorded, and a quick check of the Medicines Information View in MyHR established this. ${ }^{11}$ There it was - problem solved in a few seconds!

A few weeks later the same patient became ill at his daughter's house, some hundreds of kilometres from home. On reviewing his latest pathology result, I noted a $30 \%$ reduction in his already precarious estimated glomerular filtration rate and contacted the patient by phone. At present, phone calls are not funded, but funded 'enhanced access or other than face-to-face communication' is a key feature of 'Health Care Homes', as noted in the PHCAG report. ${ }^{2}$

It was clear from the phone conversation that the fall in renal function was not caused by a side effect of the new drug - it was a bout of gastrointestinal disturbance that triggered the acute-onchronic renal failure. I discovered that the patient had already been unwell for five days and had not been able to eat anything for the past three days. He had stopped injecting insulin when he stopped eating, but was regularly testing his blood sugar and reported that he was quite lightheaded when upright.

I referred him to the local emergency department for assessment and stabilisation. The emergency physician was able to access the patient's MyHR and check the most recent shared health summary. That summary comprised current medication (including his new medication in the most recent update), medical history and results of recent echocardiograms. Using secure messaging, I was able to send copies of the most recent electrolytes and key pathology results, a graph of his 'in surgery' seated blood pressures over the past few years and recent letters from his endocrinologist and cardiologist. This information vastly increased the emergency physician's knowledge of his background and current medical status, facilitating his emergency care.

In 2018, more and more public and private pathology, and increasing volumes of imaging results, are progressively becoming available directly from MyHR, making them rapidly accessible to new treating practitioners.
Emergency physicians and GPs trying to treat complex patients have been routinely 'flying blind' when these patients have needed their care and expertise. That is clearly about to change. The search time for information not directly sent to all of the patient's healthcare providers is also about to change. Through harmonisation and standardisation of data sets and improved interconnectivity, the information silos referred to in the introduction will progressively be broken down.

With the increasing availability of digital information to support integrated healthcare, funders are obliged to ensure that providers are sufficiently supported to achieve and maintain reliable access to these data sets, with appropriate hardware and up-to-date software applications. As indicated above, the availability of that information directly to our patients will increase their engagement in their own healthcare, altogether leading to better outcomes and a more efficient system.

Finally, my view of my role in recording medical records has changed. When I graduated, my medical notes were an aide-memoire to help me treat my patients. When I joined a group practice, I realised that my notes helped my colleagues and me treat our patients. Since computerisation, my notes and health summaries have helped me to write better referrals so that colleagues outside my practice can assist me in treating patients more effectively. Now that I can share an up-to-date health summary on MyHR, I realise that my notes can help my patients to achieve better outcomes from the health system, even when I am not directly involved.

Now that is 21 st century.

Steve Hambleton MBBS, FAMA, FRACGP (Hon), FAICD, Adjunct Professor, University of Queensland; Co-Chair My Health Record Expansion Program Steering Committee, Australian Digital Health Agency; Board Member, Digital Health CRC Limited. stevejhambleton@gmail.com

Competing interests: $\mathrm{SH}$ is the Co-Chair of the My Health Record Expansion Program Steering Committee, Clinical Programs and Clinical Reference Group Australian Digital Health Agency. He was also a past chair of NEHTA and member of PCEHR Review Advisory Group. 
Funding: $\mathrm{SH}$ is funded by Australian Digital Health Agency on the above committees.

Provenance and peer review: Commissioned, externally peer reviewed.

\section{References}

1. The Treasury. Intergenerational Report. Canberra: Commonwealth of Australia, 2011. Available at http://archive.treasury.gov.au/igr [Accessed 1 June 2018].

2. Department of Health. Primary Health Care Advisory Group final report. Better outcomes for people with chronic and complex health conditions. Canberra: Commonwealth of Australia, 2016.

3. Australian Digital Health Agency. Australia's national digital health strategy. NSW: Australian Digital Health Agency, 2018. Available at www.digitalhealth.gov.au/about-the-agency/ publications/australias-national-digital-healthstrategy [Accessed 1 June 2018].

4. Srinivasan U, Rao S, Ramachandran D, Jonas D. Flying blind: Australian consumers and digital health, Volume 1: Australian health data series. Sydney: Health Market Quality Research Program, CMCRC, 2016.
5. Australian Commission on Safety and Quality in Health Care. Annual report 2014/15. Sydney: ACSQHC, 2015.

6. Australian Digital Health Agency. MyEHR to national eHealth record transition impact evaluation. NSW: Australian Digital Health Agency, 2015. Available at www.digitalhealth.gov. au/news-and-events/news/myehr-to-nationalehealth-record-transition-impact-evaluation [Accessed 19 May 2018].

7. My Health Record. View my record using an app. NSW: My Health Record, 2018. Available at www. myhealthrecord.gov.au/for-you-your-family/ howtos/view-my-record-using-app [Accessed 1 June 2018].

8. Greene J, Hibbard JH. Why does patient activation matter? An examination of the relationships between patient activation and health-related outcomes. J Gen Intern Med 2012;27(5):520-26. doi: 10.1007/s11606-011-1931-2.

9. SingHealth. CyberAttack on SingHealth IT system - Information for Sing Health patients. Singapore: SingHealth, 2018. Available at www. singhealth.com.sg/AboutSingHealth/Pages/ datasecuritycheck.aspx [Accessed 27 June 2018].

10. My Health Record. Set privacy and security controls. NSW: My Health Record, 2018. Available at www.myhealthrecord.gov.au/for-you-yourfamily/howtos/set-privacy-and-security-controls [Accessed 27 June 2018].

11. My Health Record. Medicines information view. NSW: My Health Record, 2018. Available at www.myhealthrecord.gov.au/for-healthcareprofessionals/medicines-information-view [Accessed 1 June 2018]. 